PoS $\quad \begin{aligned} & \text { PROCEEDINGS } \\ & \text { OF SCIENCE }\end{aligned}$

\title{
Search for electroweak production of supersymmetry at CMS
}

\author{
Miaoyuan Liu on behalf of the CMS Collaboration*t \\ Fermi National Accelerator Lab. (US) \\ E-mail: mliuefnal.gov
}

We report on searches for supersymmetry via pair production of partners of electroweak gauge and Higgs bosons. The searches use proton-proton collision data recorded in 2016 by the CMS experiment at the LHC. The results are interpreted in terms of several simplified models of supersymmetry.

EPS-HEP 2017, European Physical Society conference on High Energy Physics 5-12 July 2017

Venice, Italy

* Speaker.

${ }^{\dagger}$ A footnote may follow. 


\section{Introduction}

Supersymmetry (SUSY) is an appealing extension of the standard model (SM) of particle physics. It proposes a new symmetry such that each boson (fermion) in the SM has a fermion (boson) superpartner. SUSY can potentially address several of the open questions in the SM, also provides potential candidates for dark matter. Strongly produced SUSY have been studied extensively with the LHC data, setting stringent limits on its production, while electroweakly produced SUSY is less constrained due to its lower production rate.

In the minimal supersymmetric extension of the standard model, the following gauginos exist in the electroweak sector: The gravitino is the supersymmetric partner of the graviton. The winos are the superpartners of the $\mathrm{W}$ bosons of the $\mathrm{SU}(2) \mathrm{L}$ gauge fields. The bino is the superpartner of the U(1) gauge field corresponding to weak hypercharge. Gauginos mix and form mass eigenstates which are named charginos and neutralinos.

Most searches for SUSY at the LHC additionally assume R-parity conservation. This implies that electroweak SUSY particles are produced in pairs and decay, either directly or via a cascade, to the stable lightest SUSY particle, or LSP. In the models described here, the LSP is either the least massive neutralino (Wino models) or the gravitino (Higgsino models). Depending on the SUSY model parameters, SM fermions, gauge bosons and Higgs bosons can all be produced in EW SUSY particle cascades, producing a wide variety of potential experimental signatures. A large spectrum of signatures have been studied using the $36 \mathrm{fb}^{-1} \mathrm{pp}$ data in CMS [1] and the search results are combined to reach the best sensitivity of each SUSY senario [2]. Searches for Wino models are described in Section 2 and a combined result of searches for Higgsino models is presented in Section 3. The searches of sleptons are of special interest in explaining a dark matter relic density consistent with cosmological observations. Results of a dedicated search for evidence of stau production are presented in Section 4.

\section{Searches for Wino models}

In Wino models, gaugino pairs can decay via sleptons or gauge bosons when sleptons are heavy. Early electroweak SUSY searches focus on the production of chargino-neutralino because of its large production cross section.

\subsection{Search with two same-sign leptons and three or more leptons.}

Final states of multiple leptons are sensitive to various SUSY scenarios. Decays of a charginoneutralino pair through sleptons, as shown in Fig. 1(left), naturally result in a final state of multiple leptons. Decays to a diboson pair can also produce multiple leptons if the diboson pair decays leptonically. One example is shown in Fig. 1(right), a three lepton final state can occur when the chargino decays to a $\mathrm{W}$ boson and the LSP and the neutralino decays to a $\mathrm{Z}$ boson and the LSP, with $\mathrm{W}$ and $\mathrm{Z}$ boson then decaying leptonically. A search using events with two same-sign light flavor $(\mathrm{e} / \mu)$ or at least three leptons with additional requirement on missing transverse energy $\left(p_{\mathrm{T}}^{\text {miss }}\right)$ due to LSP is presented in [3]. Events are further categorized using the mass of opposite-sign sameflavor lepton pairs and transverse mass formed by the third lepton as well as the missing transverse energy. Since the kinematic phase space can be very different in different targeted models, an 
inclusive search with 158 signal regions is designed to optimize the coverage of various SUSY scenarios. For example, exclusive missing transverse momentum regions are necessary in order to cover signatures with large and small mass-splitting between chargino and neutralino as shown in Fig 2.
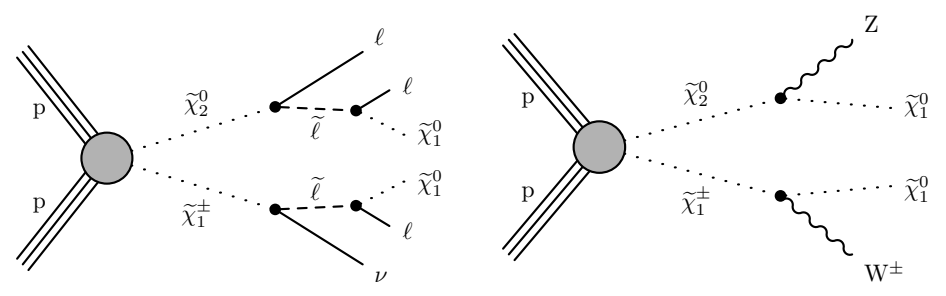

Figure 1: Left: Chargino and neutralino pair production with decays mediated by sleptons. Right: Chargino and neutralino pair production with the chargino decaying to a W boson and the LSP and the neutralino decaying to a $\mathrm{Z}$ boson [3].

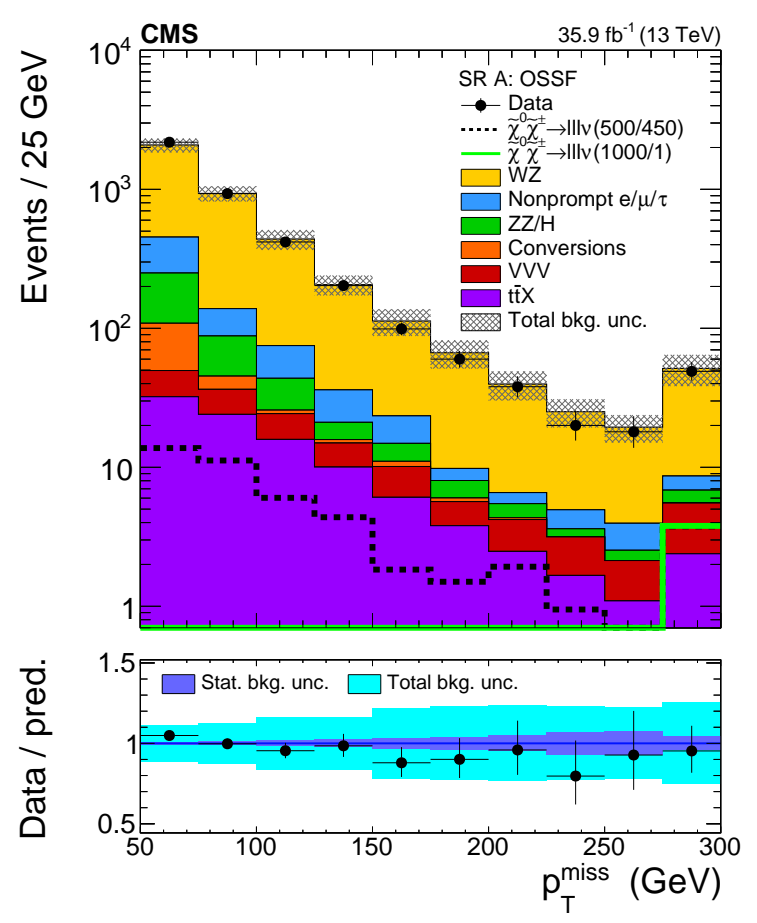

Figure 2: Distribution of the $p_{\mathrm{T}}^{\text {miss }}$ in SRA defined in [3]. Two signal mass points in the flavor-democratic model, in which the chargino and neutralino decay via sleptons or sneutrinos to all lepton flavors with the same branching fraction, are displayed for illustration [3].

\subsection{Search with one lepton plus two $b$ jets.}

Chargino and neutralino pair production yields a $\mathrm{W}$ and a $\mathrm{H}$ boson if the neutralino decays to $\mathrm{H}$ and the LSP with $100 \%$ branching fraction as shown in Fig. 3. A search is performed in the one lepton plus $b \bar{b}$ final state to target cases where $\mathrm{W}$ boson decays leptonically and higgs boson decays 
to $b \bar{b}$ [4]. This final state profits from the large branching fraction of $h \rightarrow b \bar{b}$ decay, therefore is the most sensitive channel to $\mathrm{WH}$ production.

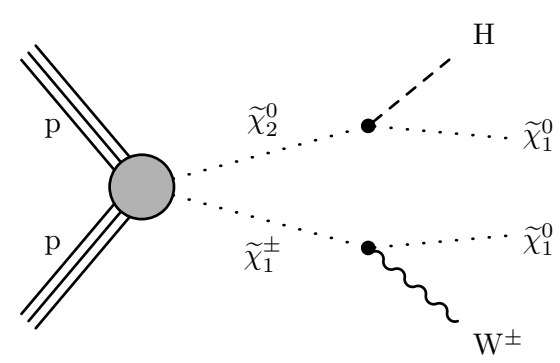

Figure 3: Diagram corresponding to the SUSY simplified model targeted by one lepton plus $b \bar{b}$, i.e., chargino-neutralino production, with the chargino decaying to a W boson and an LSP, while the heavier neutralino decays to a Higgs boson and an LSP [4].

This search selects events with exactly one charged lepton, exactly two b jets, and large $p_{\mathrm{T}}^{\text {miss }}$. The invariant mass of the two $\mathrm{b}$ jets is required to be consistent with the mass of the $\mathrm{H}$ boson. Additional kinematic variables are used to suppress backgrounds which predominantly come from dileptonic decays in $t \bar{t}$ production [4]. Two exclusive signal regions are defined based on $p_{\mathrm{T}}^{\text {miss }}: 125$ $\leq p_{\mathrm{T}}^{\text {miss }}<200 \mathrm{GeV}$ and $p_{\mathrm{T}}^{\text {miss }} \geq 200 \mathrm{GeV}$. The SM backgrounds are predicted using MC simulation, with the predictions validated in data control regions distinct from the signal region. Figure 4 shows the distributions of $M_{\mathrm{b} \bar{b}}$ in data compared with the SM background prediction after all signal region requirements except the $M_{\mathrm{b} \bar{b}}$ selection. No significant deviations from the predictions are observed.
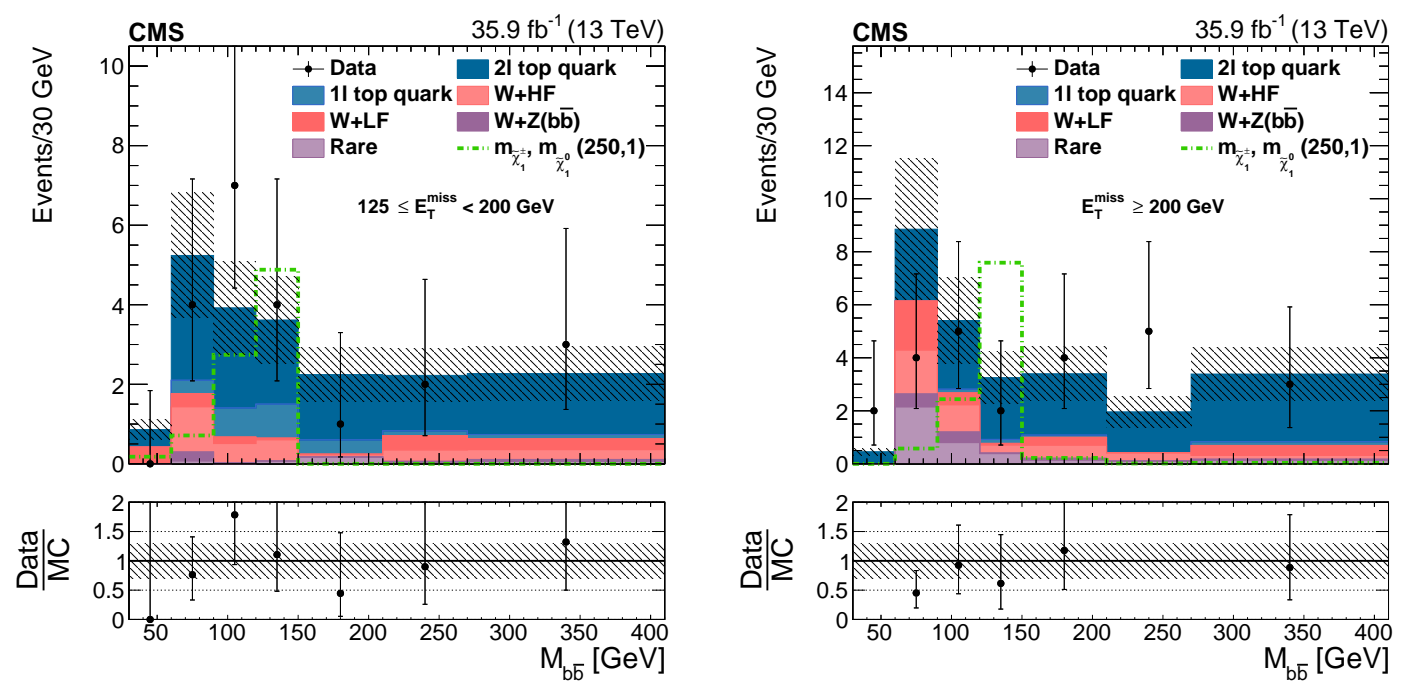

Figure 4: Distributions in $M_{\mathrm{b} \overline{\mathrm{b}}}$ after all signal region kinematic requirements for the two exclusive $p_{\mathrm{T}}^{\text {miss }}$ bins (left: $125 \leq p_{\mathrm{T}}^{\text {miss }}<200 \mathrm{GeV}$, right: $p_{\mathrm{T}}^{\text {miss }} \geq 200 \mathrm{GeV}$ ). The signal region is $90 \leq M_{\mathrm{b} \bar{b}} \leq 150 \mathrm{GeV}$. The hatched band shows the total uncertainty in the background prediction, including statistical and systematic components. The expected signal distribution for a reference SUSY model is overlaid as an open histogram, and the legend (on the last line) gives the masses in $\mathrm{GeV}$ [4]. 


\subsection{Search with opposite sign dilepton final state associated with $p_{\mathrm{T}}^{\text {miss }}$.}

This search selects events with exactly two opposite-sign, same-flavor (OSSF) leptons (ee or $\mu \mu$ ), at least two hadronic jets, and large $p_{\mathrm{T}}^{\text {miss }}$, targeting the $\mathrm{WZ}, \mathrm{ZZ}$, and $\mathrm{ZH}$ topologies [5]. Such a signature can be produced if the $\mathrm{Z}$ boson decay hadronically or the $\mathrm{H}$ bosons decays to $b \bar{b}$, tagged with a $\mathrm{Z}$ boson decay leptonically. Therefore the search is performed in both regions with or without $b$ jets [5].

\subsection{Combined results for WZ/WH}

The results of the searches presented above are interpreted using the simplified models and are combined in [2]. Figure 5 (left) shows the observed and expected limit contours for each of the individual analyses considered in the combination, and Fig. 5(right) shows the results from the combination for all three topologies considered. The opposite-sign dilepton analysis generally gives the best sensitivity for large values of $\Delta m=m_{\widetilde{\chi}_{2}^{0}}-m_{\widetilde{\chi}_{1}^{0}}$. The multi-lepton search provides the best sensitivity at intermediate values of $\Delta m$, including the region where $\Delta m \approx m_{Z}$. For a massless LSP $\widetilde{\chi}_{1}^{0}$, the combined result gives an observed (expected) limit in $m_{\widetilde{\chi}_{1}^{ \pm}}$of about 650 (570) GeV for the $\mathrm{WZ}$ topology, 480 (455) GeV for the $\mathrm{WH}$ topology, and 535 (440) $\mathrm{GeV}$ for the mixed topology. The combination also excludes intermediate mass values that were not excluded by individual analyses, including $m_{\widetilde{\chi}_{1}^{ \pm}}$values between 180 and $240 \mathrm{GeV}$ for a massless LSP in the WH topology.
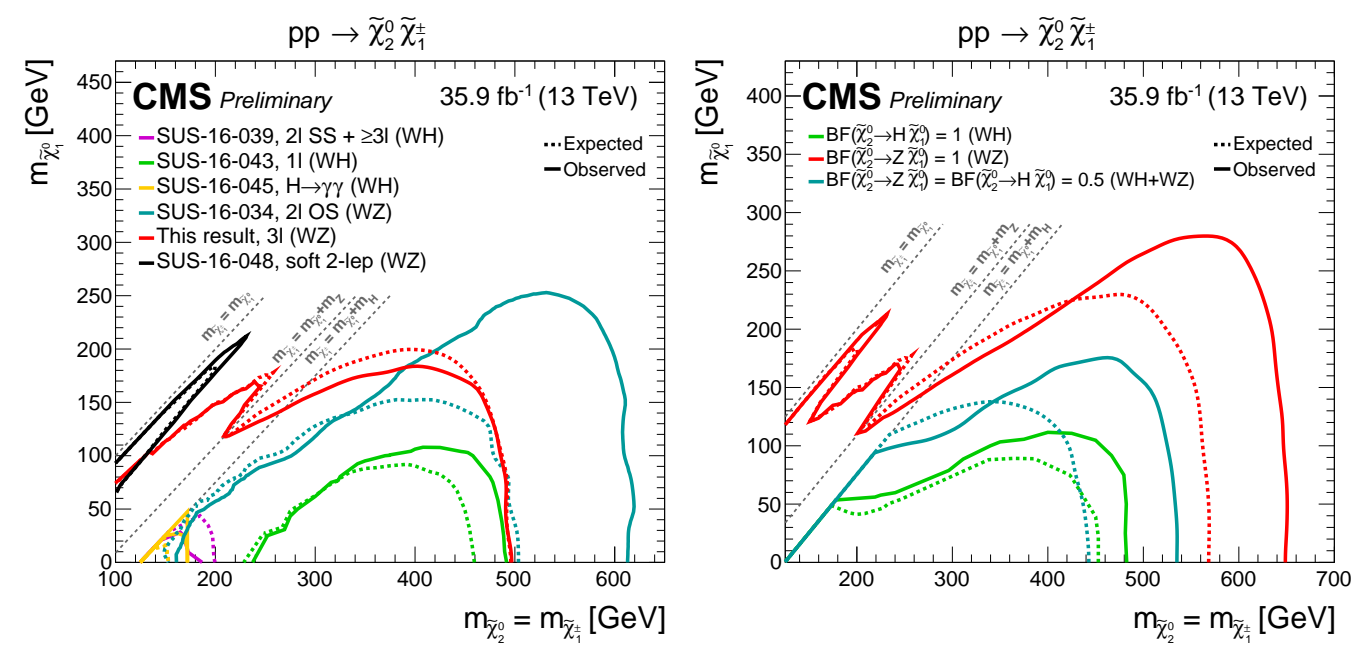

Figure 5: Exclusion contours at 95\% CL in the plane of $m_{\widetilde{\chi}_{1}^{ \pm}}$and $m_{\widetilde{\chi}_{1}^{0}}$ for the models of $\widetilde{\chi}_{1}^{ \pm} \widetilde{\chi}_{2}^{0}$ production (left) for the individual analyses and (right) for the combination of analyses. The decay modes assumed for each contour are given in the legends [2].

\section{Searches for Higgsino models}

In gauge-mediated-supersymmetry-breaking (GMSB) models, gravitino is the LSP and the lightest neutralino is the next-to-lightest SUSY particle (NLSP). As couplings to the gravitinos are very weak, SUSY particles generally cascade to the NLSP. The NLSP then decays to its SM 
partner and a gravitino, and thus the nature of the NLSP largely defines the observable signature. When light chargino, heavier neutralino and lightest neutralino are nearly mass degenerate, the NLSP pair production is enhanced by decays of chargino/heavier neutralino to lighter neutralino. The neutralinos are mixtures of bino, wino and higgsino states, and therefore a neutralino NLSP will produce a $\mathrm{Z}$ boson or a Higgs boson when it decays. Various signatures were searched for depending on the branching ratio of NLSP to the $\mathrm{H}$ boson as well as the decay mode of $\mathrm{Z} / \mathrm{H}$ bosons. For example, in the HH channel, the Higgs boson decays primarily to $b \bar{b}$, and so the dominant final state is $4 \mathrm{~b}+p_{\mathrm{T}}^{\text {miss }}$. This is probed by a search looking for 4 -jet events with multiple b-tagged jets and significant $p_{\mathrm{T}}^{\text {miss }}$ [6]. Figure 6 shows the combined results of searches for Higgsino models. The limits are presented as a function of Higgsino mass and the branching ratio of decaying to the $\mathrm{H}$ boson and shown for each individual analysis as well as combined result. Higgsino mass is excluded up 650 to $700 \mathrm{GeV}$, with small dependence on the branching ratio of decays to the $\mathrm{H}$ boson.

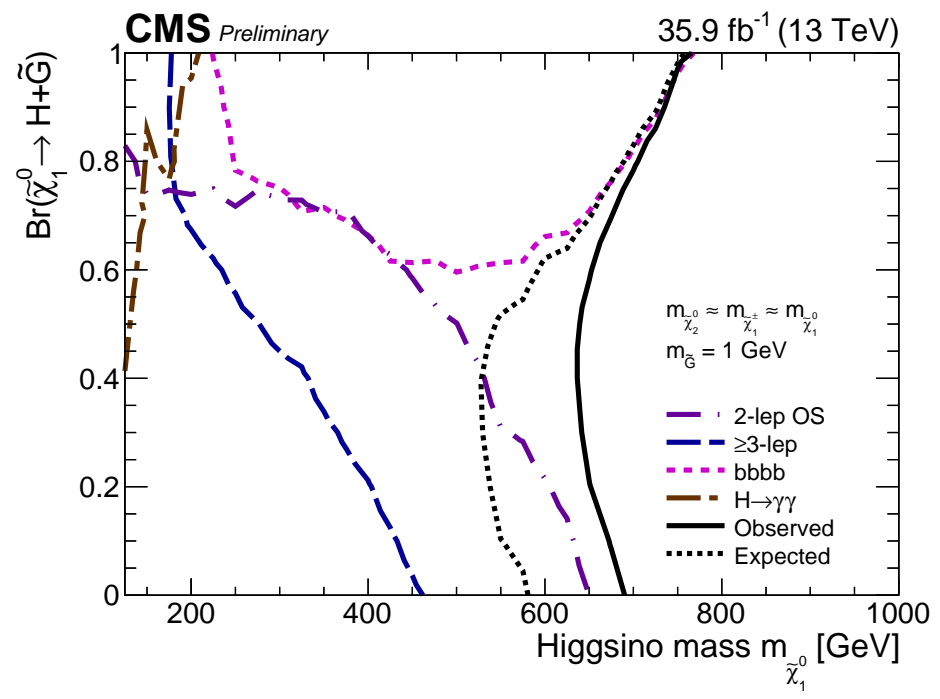

Figure 6: Observed exclusion contours at the $95 \% \mathrm{CL}$ in the plane of $m_{\widetilde{\chi}_{1}^{0}}$ and $\mathscr{B}\left(\widetilde{\chi}_{1}^{0} \rightarrow H \widetilde{G}\right)$ for the model of $\widetilde{\chi}_{1}^{0} \widetilde{\chi}_{1}^{0}$ production for each individual analysis compared with the combination. The $4 b$ search drives the exclusion at large values of $\mathscr{B}\left(\widetilde{\chi}_{1}^{0} \rightarrow H \widetilde{G}\right)$ while the on- $Z$ dilepton and multilepton searches are competing at lower values of $\mathscr{B}\left(\widetilde{\chi}_{1}^{0} \rightarrow H \widetilde{G}\right)[2]$.

\section{Searches for direct slepton production}

In thermal cosmology scenarios with a bino LSP, the annihilation cross section of dark matter particles would be too small, leading to an overabundance of dark matter compared to the current constraints from cosmological measurements. The existence of another, nearly mass-degenerate, SUSY particle that could co-annihilate with the dark matter particle would reduce the amount of dark matter to a level consistent with cosmological observations. Co-annihilation with a light stau that has a small mass splitting with a bino LSP leads to a dark matter relic density consistent with cosmological observations. Therefore it is important to search for evidence of stau production at 
the LHC. A search targeting a simplified model with direct stau pair production has been performed using the 2016 data collected by CMS, where the staus decay to a tau lepton and a neutralino which is considered to be the LSP. We focus on the final state where the tau leptons decay hadronically. No excess above the SM is observed in the signal regions considered. The results are interpreted for three different helicity scenarios. This analysis is most sensitive to a scenario with left-handed stau of around $125 \mathrm{GeV}$ and a massless LSP, where we exclude 1.5 times the expected SUSY cross-section [7].

\section{Conclusions}

An inclusive set of searches of electroweak SUSY production have been performed using the $36 \mathrm{fb}^{-1}$ pp collision data collected by CMS in 2016. Various SUSY scenarios are considered in these searches and no hint of electroweakly produced SUSY is found. Analysis results have been combined to cover SUSY parameter space gaps, setting the best constraints on electroweak SUSY production in many models.

\section{References}

[1] CMS Collaboration, The CMS experiment at the CERN LHC, JINST 3 (2008) S08004, doi:10.1088/1748-0221/3/08/S08004.

[2] CMS Collaboration, Combined search for electroweak production of charginos and neutralinos in $p p$ collisions at $\sqrt{s}=13 \mathrm{TeV}$, CMS-PAS-SUS-17-004,

[http://cds.cern.ch/record/2273907]

[3] CMS Collaboration, Search for electroweak production of charginos and neutralinos in multilepton final states in proton-proton collisions at $\sqrt{s}=13 \mathrm{TeV}$, [arXiv: 1709.05406$]$

[4] CMS Collaboration, Search for electroweak production of charginos and neutralinos in WH events in proton-proton collisions at $\sqrt{s}=13 \mathrm{TeV},[\operatorname{arXiv}: 1706.09933 \mathrm{v} 1]$

[5] CMS Collaboration, Search for new phenomena in final states with two opposite-charge, same-flavor leptons, jets, and missing transverse momentum in pp collisions at $\sqrt{s}=13 \mathrm{TeV}$, [arXiv:1709.08908]

[6] CMS Collaboration, Search for higgsino pair production in pp collisions at $\sqrt{s}=13 \mathrm{TeV}$ in final states with large missing transverse momentum and two Higgs bosons decaying via $H \rightarrow b \bar{b}$, [arXiv:1709.04896]

[7] CMS Collaboration, Search for pair production of tau sleptons in $\sqrt{s}=13 \mathrm{TeV}$ pp collisions in the all-hadronic final state, CMS-PAS-SUS-17-003, [http: //cds . cern.ch/record/2273395] 